\title{
MODELING DESERT DUST EXPOSURE IN EPIDEMIOLOGIC SHORT-TERM HEALTH EFFECTS STUDIES
}

Aurelio Tobías (1), Massimo Stafoggia (2)

1. Institute of Environmental Assessment and Water Research (IDAEA), Spanish Council for Scientific Research (CSIC), Barcelona, Spain.

2. Department of Epidemiology, Lazio Regional Health Service/ASL Roma 1, Rome, Italy.

Supplementary Tables

eTable 1. Descriptive characteristics of Rome, for the study period $2005-2015$........2

Supplementary Figures

eFigure 1. Time series distribution of daily mortality (top) and $\mathrm{PM}_{10}$ concentrations (bottom) during dust and non-dust days in Rome, in 2007 ..............................

eFigure 2. Time series distribution of desert (top) and non-desert $\mathrm{PM}_{10}$ loads (bottom) during dust and non-dust days in Rome, in 2007 
eTable 1. Descriptive characteristics of Rome, for the study period 2005-2015.

\begin{tabular}{lrlrrrr}
\hline & Mean & $(\mathbf{s d})$ & Min. & $\mathbf{1 0}^{\text {th }}$ pct & $\mathbf{9 0}^{\text {th }}$ pct & Max. \\
\hline All days $(\mathrm{n}=4017)$ & & & & & & \\
Mortality & 58.8 & $(10.0)$ & 25.0 & 47.0 & 72.0 & 105.0 \\
$\mathrm{PM}_{10}\left(\mu \mathrm{g} / \mathrm{m}^{3}\right)$ & 31.8 & $(13.9)$ & 4.0 & 17.0 & 51.0 & 160.0 \\
$\quad$ Desert & 1.5 & $(5.7)$ & 0.0 & 0.0 & 5.0 & 129.7 \\
$\quad$ Non-desert & 30.3 & $(13.3)$ & 0.0 & 16.7 & 48.3 & 98.9 \\
Temperature $\left({ }^{\circ} \mathrm{C}\right)$ & 15.9 & $(7.0)$ & -1.0 & 6.8 & 25.7 & 32.5 \\
& & & & & & \\
Dust days $(\mathrm{n}=575)$ & & & & & & \\
Mortality & 58.2 & $(9.6)$ & 25.0 & 46.0 & 71.0 & 90.0 \\
PM10 $\left(\mu \mathrm{g} / \mathrm{m}^{3}\right)$ & 36.7 & $(14.2)$ & 11.0 & 22.8 & 53.5 & 160.0 \\
$\quad$ Desert & 10.7 & $(11.2)$ & 0.0 & 1.3 & 22.0 & 129.7 \\
$\quad$ Non-desert & 25.9 & $(9.1)$ & 0.0 & 17.0 & 37.8 & 80.0 \\
Temperature $\left({ }^{\circ} \mathrm{C}\right)$ & 20.2 & $(5.7)$ & 5.9 & 12.3 & 27.4 & 31.0 \\
Non-dust days $(\mathrm{n}=3420)$ & & & & & & \\
Mortality & 59.0 & $(10.1)$ & 30.0 & 47.0 & 72.0 & 105.0 \\
PM $10\left(\mu \mathrm{g} / \mathrm{m}^{3}\right)$ & 31.0 & $(13.7)$ & 4.0 & 16.6 & 50.3 & 98.9 \\
$\quad$ Desert & 0.0 & $(0.0)$ & 0.0 & 0.0 & 0.0 & 0.0 \\
$\quad$ Non-desert & 31.0 & $(13.7)$ & 4.0 & 16.6 & 50.3 & 98.9 \\
Temperature $\left({ }^{\circ} \mathrm{C}\right)$ & 15.1 & $(6.9)$ & -1.0 & 6.3 & 25.0 & 32.5 \\
\hline
\end{tabular}

SD indicates standard deviation. 
eFigure 1. Time series distribution of daily mortality (top) and $\mathrm{PM}_{10}$ concentrations (bottom) during dust and non-dust days in Rome, in 2007.
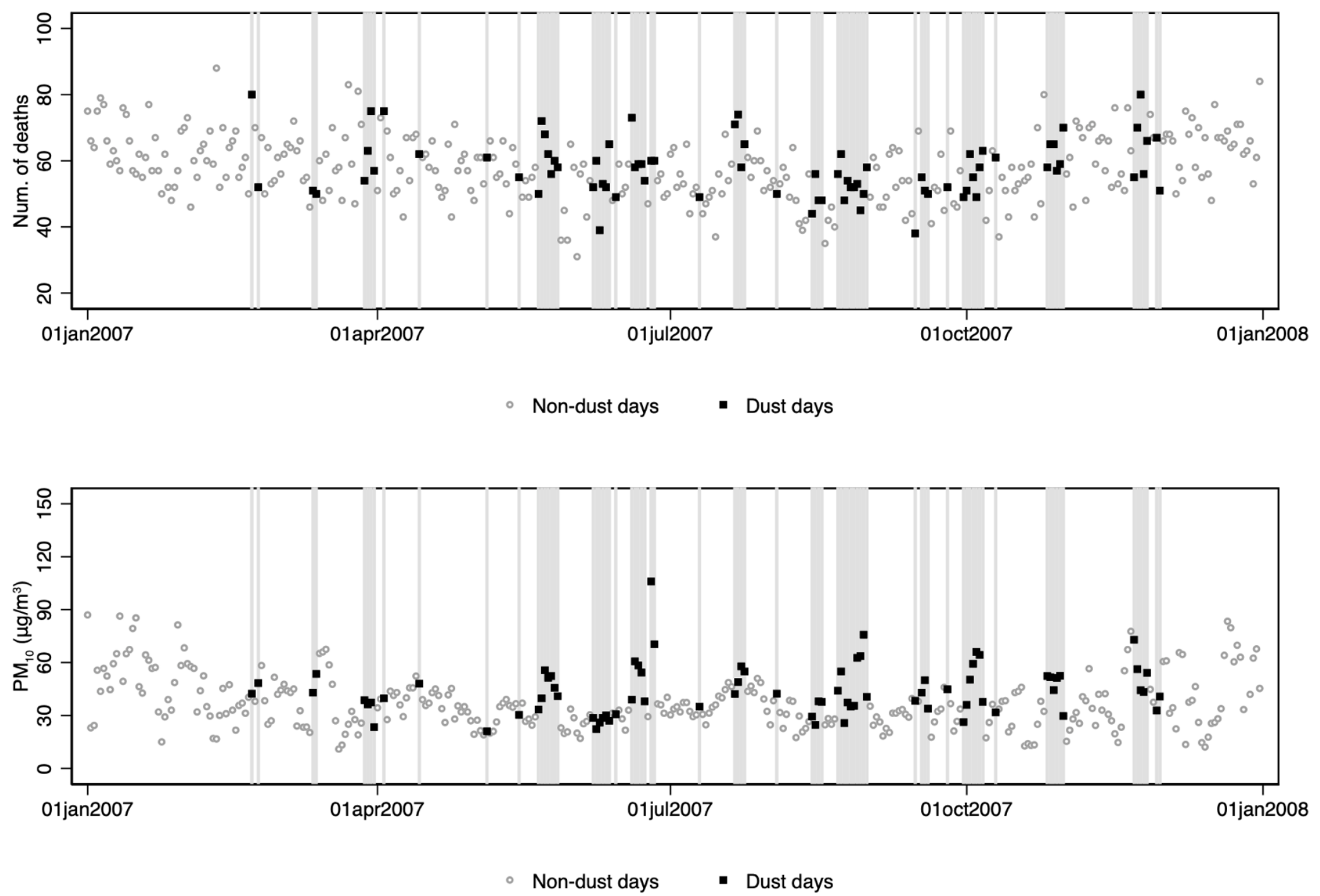
eFigure 2. Time series distribution of desert (top) and non-desert $\mathrm{PM}_{10}$ loads (bottom) during dust and non-dust days in Rome, in 2007.
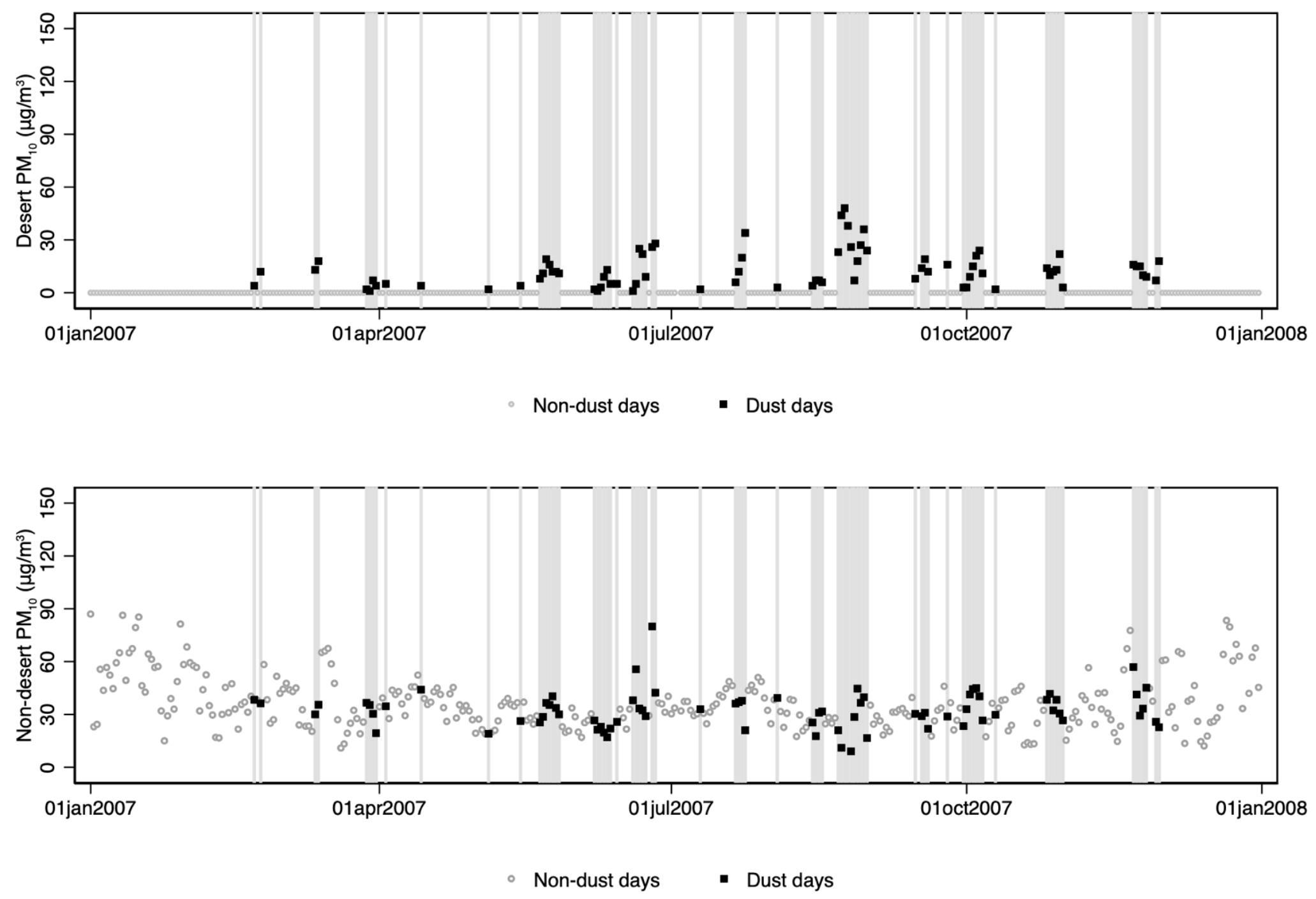